\title{
Gradhiva
}

GRADHIV

Revue d'anthropologie et d'histoire des arts

$30 \mid 2019$

Précieux

\section{Romain Bertrand, Le Détail du monde : l'art perdu de la description de la nature}

Paris, Seuil, coll. «L'univers historique », 2019

\section{Frédéric Keck}

\section{(2) OpenEdition}

\section{Journals}

Édition électronique

URL : http://journals.openedition.org/gradhiva/4793

DOI : $10.4000 /$ gradhiva.4793

ISSN : 1760-849X

\section{Éditeur}

Musée du quai Branly Jacques Chirac

Édition imprimée

Date de publication : 4 décembre 2019

Pagination : 156

ISBN : 978-2-35744-113-2

ISSN : 0764-8928

\section{Référence électronique}

Frédéric Keck, « Romain Bertrand, Le Détail du monde : l'art perdu de la description de la nature », Gradhiva [En ligne], 30 | 2019, mis en ligne le 31 mars 2021, consulté le 01 avril 2021. URL : http:// journals.openedition.org/gradhiva/4793; DOI : https://doi.org/10.4000/gradhiva.4793

Ce document a été généré automatiquement le 1 avril 2021.

(c) musée du quai Branly 


\section{Romain Bertrand, Le Détail du} monde : l'art perdu de la description de la nature

Paris, Seuil, coll. « L'univers historique », 2019

Frédéric Keck

\section{RÉFÉRENCE}

Romain Bertrand, Le Détail du monde : l'art perdu de la description de la nature. Paris, Seuil, coll. « L'univers historique », 2019, 278 p. 


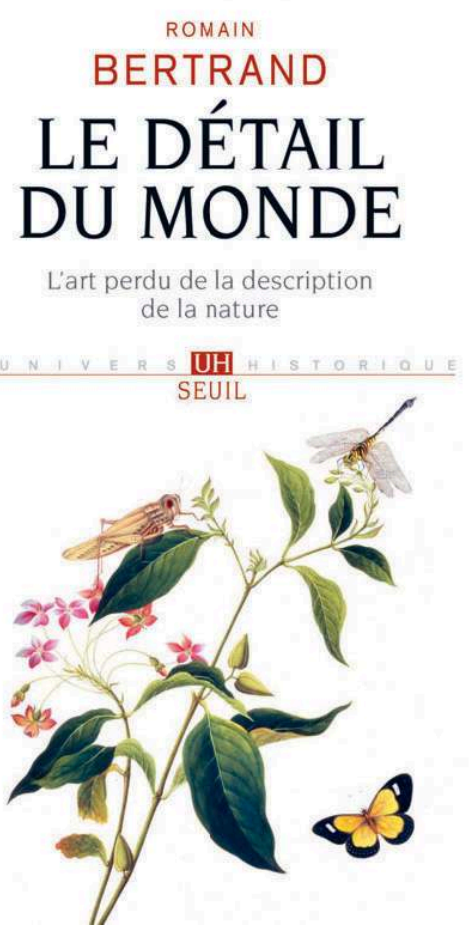

Romain Bertrand, connu pour ses histoires connectées de l'Asie du Sud-Est ${ }^{1}$, se livre ici à une "rêverie » depuis les côtes de Bornéo, où il a récemment consulté des archives. Au seuil d'un nouvel ouvrage d'histoire du monde malais, il évoque les figures qui l'ont précédé dans la contemplation d' " un univers à l'agonie, un monde à claire-voie, dont les surfaces s'étiolent, de toutes parts livrées aux outrages de l'entaille» (p. 12). Le premier est Alfred Russel Wallace (1823-1913), auteur de The Malay Archipelago en 1869, collecteur passionné de coléoptères, qui voyagea dans l'Asie-Pacifique pour en décrire les espèces - une ligne géographique porte encore son nom, séparant les espèces asiatiques de celles du monde austral - et formula en même temps que Charles Darwin la théorie de l'évolution des espèces par sélection naturelle. Le second est Tom Harrisson (1911-1976), qui appliqua à l'observation des hommes les méthodes qu'il avait d'abord apprises dans l'observation des oiseaux, combattit l'armée japonaise pendant la Seconde Guerre mondiale et dirigea pendant près de trente ans le musée du Sarawak. Entre ces deux figures centrales de l'ouvrage, qui finirent leurs carrières scientifiques dans la marginalité, Romain Bertrand évoque les grands naturalistes européens pour tenter de retrouver l'unité d'un monde décrit dans la variété de ses surfaces, comme si le langage pouvait en colmater les entailles.

Ce rêve naturaliste est rapporté par Romain Bertrand à l'entreprise d'Alexander von Humboldt (1769-1859), interlocuteur de Goethe et de Schiller à Iéna, dont le récit de voyage en Amazonie fut une référence pour Darwin autant que pour Wallace. Une gravure du volcan Chimborazo, situé au centre de l'Équateur, revient plusieurs fois au cours de l'ouvrage: Humboldt y rapporte sur la moitié gauche les entités visibles plantes et roches - et sur la moitié droite leurs noms savants. « Loin de combler le fossé entre les mots et les choses, la gravure le rend plus visible, plus sensible que jamais. Mais à l'instar de l'art japonais du kintsugi, qui use de pâte d'or pour restaurer les 
porcelaines cassées, il s'agit moins d'abolir la brisure que de la conjurer, de déjouer son péril en l'exposant. » (p. 49) Cette faille entre les mots et les choses, si elle peut être comblée pour les roches et les plantes, devient en effet béante pour les animaux humains et non humains ; c'est pourquoi les premiers collecteurs se passionnent pour les insectes, dont l'abondance et la disponibilité permettent de les tuer sans remords et de les exposer en série.

3 En effet, toute une équipée de traqueurs et de chasseurs indigènes accompagne les naturalistes avant la préparation des taxidermies pour les envoyer dans les métropoles. Si le premier chapitre du livre montre le lien entre la collecte des coléoptères et la théorie de l'évolution des espèces, le deuxième souligne que la chasse est la condition de la mise en scène de la nature, à travers l'alliance improbable entre le peintre Louis Tinayre, fils de communards, et le prince Albert de Monaco, souverain conservateur passionné de chasse. La Carrière d'un navigateur (1914), illustrée par Tinayre, raconte les exploits de chasse du prince de Monaco en Méditerranée, en mer du Nord et dans l'Arctique. Dans une scène saisissante, un matelot décapite une tortue de mer, et le peintre plonge son pinceau dans la bile qui s'en écoule, comme s'il pouvait peindre la nature avec les couleurs qu'il lui arrache. Cette scène détermine la réflexion menée dans le deuxième chapitre, intitulé «Le bleu des choses ", sur la tentative de décrire la nature telle un spectre de couleurs, abandonnant ainsi le rêve de Humboldt, partagé par Wallace et Darwin, d'une science à la fois orientée vers des principes théoriques et attentive à la diversité des êtres. Romain Bertrand critique cet aplatissement de la nature chez Ernst Haeckel (1834-1919), disciple allemand de Darwin : «Les radiolaires et les méduses des Formes artistiques de la nature n'ont plus aucune histoire individuelle : ce sont des spectres sans visage.» (p. 145) Le moment de la microphotographie est analysé comme une trahison de la surface des êtres au nom d'une fausse profondeur présentée sur des lamelles : «Par un glissement de sens macabre, le détail des êtres en vient à signifier leur débitage.» (p. 147) Le récit historique abandonne alors ce riche moment de la culture visuelle de la fin du XIX ${ }^{e}$ siècle pour passer du côté de l'écriture poétique, en citant Paul Valéry, Francis Ponge et D.H. Lawrence. Un tel basculement de la couleur vers la poésie peut surprendre, mais il résulte du diagnostic porté sur l'échec du langage à saisir une nature qui s'est dépeuplée.

Le troisième chapitre renverse alors ce diagnostic à travers une figure méconnue, celle de Tom Harrisson, ornithologue et sociologue britannique, dernier représentant du rêve de Humboldt. C'est tout le monde du birdwatching qui est évoqué dans cette partie, avec son effort pour observer la nature à distance sans prélever de spécimens, comme si les oiseaux permettaient aux hommes de rester à la surface des choses sans l'inciser. L'ornithologie repose à la fois sur une critique morale de la chasse à des fins de consommation ou d'ornementation - la Plumage League fondée en 1885 vise à bannir l'usage des plumes dans les parures de mode, ce qu'elle obtient par le Plumage Act de 1921 - et sur un effort scientifique pour voir le monde à travers le regard des oiseaux, notamment dans les travaux d'Eliot Howard, popularisés par Edward Max Nicholson et Julian Huxley. Tom Harrisson, éduqué à Harrow puis à Oxford dans ce milieu moral et intellectuel, lance en 1937 le Mass Observation Project, qui obtient le soutien du gouvernement pendant la guerre, et vise à décrire les mouvements des masses populaires britanniques avec la précision neutre des recensements de birdwatching. « La manière même de parler d'une rue comme d'un espace continûment strié de passages, livré tantôt aux cris et tantôt au silence, évoque la description d'une forêt bruissant de déplacements furtifs.» (p. 204-205) Parallèlement à cette "anthropologie de nous- 
mêmes ", Tom Harrisson effectue sur les traces de Bronislaw Malinowski des voyages au Sarawak et aux Nouvelles-Hébrides, où il décrit son immersion chez les " cannibales », bien qu'il ne parle pas les langues locales. « Ce qui, chez Tom Harrisson, réunit ornithologie et anthropologie, zoologie et sociologie, ce n'est pas l'identité des objets mais l'unité de la méthode : les indigènes et les prolétaires, à l'image des oiseaux, ne se décrivent qu'à leur insu, affairés aux tâches répétitives et d'autant plus significatives de leur survie. Il faut les observer lorsqu'ils piètent.» (p. 225) Cet éclectisme est critiqué pour son imprécision par les anthropologues de métier, tel Edmund Leach, ce qui conduit Romain Bertrand à voir en Harrisson «l'un des derniers praticiens instinctifs d'une "histoire naturelle" qui ne séparait pas radicalement l'étude des humains et celle des non-humains, non plus que l'analyse des comportements de celle des milieux » (p. 233).

5 Ce « paradigme des derniers $s^{2} »$ qui semble orienter la démonstration peut surprendre. La rêverie de l'historien semble se clore sur la nostalgie d'une nature dont le langage épouserait les surfaces en traversant les disciplines. Il serait erroné cependant de lire cet ouvrage comme une méditation mélancolique depuis le bout du monde sur l'impossibilité de rejoindre les mots et les choses, ou encore comme une libre transgression des frontières disciplinaires au nom de figures de savants marginaux. Il s'agit plutôt d'une pause au seuil d'une nouvelle exploration du monde malais pour en dessiner des orientations possibles. L'histoire, dans sa pratique systématique de l'archive, peut déjouer le diagnostic de l'historien sur l'art perdu du détail.

\section{NOTES}

1. Voir Romain Bertrand, État colonial, noblesse et nationalisme à Java: la tradition parfaite (XVII $\mathrm{XX}$ siècles). Paris, Karthala, 2005 ; L'Histoire à parts égales : récits d'une rencontre Orient-Occident (XVI ${ }^{e}$ XVII ${ }^{e}$ siècles), Paris, Seuil, 2011 ; Le Long Remords de la Conquête : Manille-Mexico-Madrid, l'affaire Diego de Ávila (1577-1580), Paris, Seuil, 2015.

2. Voir Daniel Fabre, «D'une ethnologie romantique », in Daniel Fabre et Jean-Marie Privat (dir.), Savoirs romantiques: une naissance de l'ethnologie. Nancy, Presses universitaires de Nancy, 2010 : 5-75.

\section{AUTEURS}

FRÉDÉRIC KECK

keck.fred[at]gmail.com 